\title{
Engaging with Charities On Social Media: Comparing Interaction on Facebook and Twitter
}

\author{
Christopher Phethean, Thanassis Tiropanis, and Lisa Harris \\ Web Science Institute, University of Southampton, UK, \\ \{C.J.Phethean | T.Tiropanis | L.J.Harris\} @soton.ac.uk
}

\begin{abstract}
Social media are commonly assumed to provide fruitful online communities for organisations, whereby the brand and supporterbase engage in productive, two-way conversations. For charities, this provides a unique opportunity to reach an audience for a relatively low cost, yet some remain hesitant to fully embrace these services without knowing exactly what they will receive in return. This paper reports on a study that seeks to determine the extent to which these conversations occur, and compares this phenomenon on Facebook and Twitter for a sample of UK-based charities. Focus was placed on analysing conversations as signs of developing relationships, which have previously been shown to be a key target for charities on social media. The results of this study find that while there is an expected proportion of the audience who prefer to listen rather than engage, there is strong evidence of a core group of supporters on each site who repeatedly engage. Interestingly, disparities between how this occurs on Facebook and Twitter emerge, with the results suggesting that Facebook receives more conversations in response to the charities' own posts, whereas on Twitter there is a larger observable element of unsolicited messages of people talking about the charity, which in turn produces a differing opportunity for the charity to extract value from the network. It is also found that posts containing pictures receive the highest number of responses on each site. These were a lot less common on Twitter and could therefore offer an avenue for charities to increase the frequency of responses they achieve.
\end{abstract}

Keywords: Social media, charities, marketing, communication

\section{Introduction}

Social media's popularity in the contemporary world makes it easy to think that people interacting and engaging online can transform the process of information production and dissemination and produce a constant stream of brand advocates, supporters and critics for any organisation. In an idealistic sense, analysing this at scale would allow the organisation to determine the current perception of their services, which they could then use to encourage further engagement and generate a more loyal supporter base who help to contribute to their own marketing efforts. For charities, it is essential that they know whether or not this is really possible when they are making the decision on whether to 
allocate limited funds to developing and maintaining a social presence online. A problem arises, however, in that the level of understanding around how effective these services actually are for establishing these social relationships is limited. It is easy to assume, given the constant media hype around them, that these sites breed user engagement and citizen activism. However, there are also established 'rules' to suggest that the percentage of any community online which actually engages is small [10]. If this is the case for a charity's online presence, then this could have great implications on their perception of the 'value' or 'worthiness' of social media to them as an organisation.

This paper, therefore, seeks to assess what evidence there is on social media of developing relationships between charity and supporter. This is motivated by previous work interviewing members of social media teams at various charities in the UK which found that relationship building was seen as one of the key aims and values of using social media [13]. Therefore, the goal of this paper is to show to what extent this phenomenon is actually apparent. Further contributions are made by comparing two of the largest social networks - Facebook and Twitterto examine whether there is any difference in the levels by which people appear to engage and how these each contribute to the information transformation offered by the Internet. Studies into social media frequently look at Twitter due to the ease of use of the API to collect data, yet few compare this phenomenon to what occurs on other networks. While some (e.g. [11], [15]) do explore this difference from a brand perspective, it remains an under-researched area for a topic with as much interest as social media.

Most significantly, however, this paper emphasises that statistics measuring social media interactions which may represent a developing relationship can only reveal so much about this area, and that a more holistic approach which moves away from looking solely at social media statistics is required. A qualitative analysis of some of the most engaging messages is provided to investigate the content and themes that lead to the most signs of interaction. Future directions are outlined that insist on the integration of quantitative social media statistics, qualitative social media content analysis and the views, goals and needs of the charity itself.

\section{Background}

\subsection{Relationship Building by Charities on Social Media}

For both corporations and nonprofit organisations such as charities, social media can offer an opportunity to produce a number of different outcomes. Spreading awareness of a new product or campaign, referring users to websites in order to increase traffic, generating buzz to gain media attention, and building relationships with audience members are all possibilities. Additionally each social media site offers a unique set of features that affords different types of interaction and which may make certain aims more suitable for particular sites. However, there is an equal sense that sometimes use of these sites maybe does not match their perceived value. Twitter, for example, is often presented as a great way to offer 
rapid customer service and to interact with supporters through two-way engagement. A content analysis of USA-based nonprofits' tweets instead indicates that their focus is more on sending one-way messages in order to broadcast information [19], and similar studies have also suggested a reluctance to move away from primarily information spreading behaviour [9].

A case study of the American Red Cross elicits aims for social media including the discovery of public perception, highlighting areas of improvement and generating media attention [3]. It is claimed by the authors of the study that the American Red Cross can be used as a model to follow for organisations wanting to effectively utilise social media, with an interactive two-way communicative approach essential [3]. Familiar benefits of this were listed: rapid community service and the elicitation of positive and negative feedback [3]. It must be questioned, however, how representative this feedback is if it is only sourced from a small subset of the audience who are responding. Nonetheless, interactivity on social media is said to be essential in allowing productive relationships to develop with supporters; a lack of it could potentially turn supporters away [18], and it can generally increase trust [8]. Consequently, even if the relative proportion of engaging users is low, just by showing that there is a two-way, responsive conversation could increase the trust of those users who do not with to interact online.

Interviewing members of charities who were involved in their social media presence, the current authors identified recurring themes about why social media was used, and what they hoped to get out of using it [13]. One of the most important reasons for using social media was to develop relationships with supporters, and achieving 'action' through donations was seen as a positive side-effect of doing this rather than a primary aim. There was a slight favouring towards Facebook for achieving this, especially as it provided a centralised location for people to provide support and advice- both from the charity to supporter, and supporter-to-supporter [13]. There was less clarity regarding the actual success of these sites in achieving relationship building. Accounts of favourable outcomes were given relating to the number of 'likes' content received on Facebook, which does not necessarily indicate that the users involved have a strong relationship with the charity [13].

\subsection{Online Listeners and Slacktivists}

Within any community - online or off - there will be a portion of it that does not interact or contribute, but frequently consumes the content created by others. Throughout the 1990s and early 2000s, it began to be established that for an online community, around $90 \%$ of members would fall in to this category - often labelled as 'lurkers' (for example, see [10]). [16] claims that there is a difference between "passive lurkers" and "active lurkers' who go on to use the information gathered from the online community in an offline setting, providing the beginning of an argument to suggest that the common negative perception towards these users may be undeserved. More recently, [6] discusses the stigma attached with the term 'lurker' and suggests a reconceptualisation to 'listener' instead - 
reflecting an equally important role in any online conversation as that of speaking. This also more accurately reflects the activities that many of these users will be carrying out but while a listener can be engaged in a conversation just as much as a frequent commenter, they may be missed by social media analyses looking solely for interactions. Furthermore, research on Facebook has shown that people frequently underestimate the size of the audience that is exposed to a post, as inaccurate measures such as amounts of feedback and friend counts are used, which do not reflect how many people actually listened [1].

What may be possibly more misleading when examining social media for these interactions is the phenomenon of 'slacktivism' - described in [14] as activities that are low cost and risk, and generate satisfaction in the actor. If this satisfaction is generated to replace that gained by actually doing something rather than just clicking 'like', for example - then these actions can be misinterpreted by charities whose returns will not correlate with the actions occurring on social media.

\subsection{Conversations as Indications of Relationships?}

While actions that just require 'clicking' to complete - such as liking on Facebook and retweeting on Twitter - may be looked at as being possible instances of slacktivism, other actions on these sites can reflect more meaningful signs of engagement, and may indicate a stronger relationship between charity and supporter. In earlier work by the current authors, a preliminary framework for social media measurement was presented (focusing at the time on Twitter), with retweets and replies listed as indications of engagement [12]. Previous work has provided methods of analysing retweets as a mechanism for communication and disseminating topics through the network $([2,17])$. However, while these papers show the reasons behind retweeting are varied, the replies metric appears to be a more appropriate measure for discovering strong, developed relationships: a reply requires a larger investment in terms of time and effort than simply clicking retweet, and as such [4] describes textual comments on Facebook as the highest possible level of engagement. In addition, to facilitate the two-way communication discussed above on Twitter, replies - and posts by the audience mentioning the charity that can be replied to - are essential aspects that must be analysed. Likewise, on Facebook, the reply or comment feature would showcase more than a like, and represent evidence of a strengthened relationship. In [7], the finding that 'requests and suggestions', 'expressing affect' and 'sharing' are popular intentions for participation, also suggests that textual comments will play a key role in users' interactions. In addition, overcoming users opting for the 'safest' options - those that do not provoke reactions from other members of the community, e.g. liking - should be encouraged and that improving the level of activity through conversational interactions should be sought [7]. This again suggests a significant value in conversations on social media, as they go beyond the 'easy' and 'safe' options, to show the organisation that there really is a valuable relationship present. 
For the purposes of this paper, replies and mentions on each site will be used as a representation of engagement. While retweets, likes and shares are not being focused on, the authors do not disregard their impact - indeed it is appreciated that each of these mechanisms plays a vital role in the social media marketing mix. In the current study, however, the evidence for strong relationships is sought based on the discussion above about this being a key aim for charities on social media, and that replies seem to be a viable channel to indicate this. The current study seeks to answer the following questions:

RQ1 Does either Twitter or Facebook show evidence of more sustained relationships between supporters and charity?

RQ2 Do posts by a charity on either Twitter or Facebook tend to generate more engagement than on the other?

RQ3 Do certain types of posts by a charity on either site tend to generate more or longer conversations with the audience?

\section{Methodology}

Based on the research questions devised above, a number of hypotheses are proposed. For RQ1, examining whether Twitter or Facebook creates more sustained relationships, it will be necessary to identify commenting users as discussed above to signify users who have a more developed relationship, and to determine how developed those relationships are through the intensity by which they engage. To further explore this area, and to indicate whether achieving engagement on one site could be down to the strategies carried out on that site, rather than an underlying popularity and tendency for users to engage anyway, the correlation between the scores on the two sites will be examined. This leads to the first two hypotheses:

H1a Engaging supporters for each charity will post significantly more comments on one of Facebook or Twitter than the other.

H1b If engagement is related to the overall popularity of the charity, then charities with more engaged supporters on Facebook will also have more engaged supporters on Twitter.

After testing these two hypotheses, it will be important to relate this back to the authors' previous work, which suggested that charities believed Facebook is the better platform for developing relationships [13].

Looking at RQ2, it can be seen that providing insights in to this question will help reinforce this argument, and go on to provide recommendations to charities as to which site would be worth spending more time on - or which would be better to dedicate limited resources to. As with the hypotheses for RQ1, it is expected that from the opinions shared in [13], there will be noticeable differences in the ways in which users respond to posts on each of the two networks. Whereas H1a and H1b looked at overall engagement towards the charity, RQ2 focuses on the responses to the charities' posts in order to discover the importance of the 
Table 1: Data overview

\begin{tabular}{|l|l|l|l|l|l|l|}
\hline & \multicolumn{3}{|c|}{ Facebook Data } & \multicolumn{3}{c|}{ Twitter Data } \\
\hline Charity & Likes & $\begin{array}{l}\text { Charity's } \\
\text { Root Posts }\end{array}$ & Commenters & Followers & $\begin{array}{l}\text { Charity's } \\
\text { Root Tweets }\end{array}$ & Mentioners \\
\hline DUK & 57834 & 135 & 3012 & 76808 & 820 & 11472 \\
\hline DT & 583569 & 451 & 12787 & 115687 & 1249 & 21083 \\
\hline WH & 529 & 76 & 27 & 586 & 424 & 179 \\
\hline HfH & 324490 & 269 & 7371 & 224259 & 2901 & 82154 \\
\hline JfG & 4973 & 252 & 495 & 12045 & 3818 & 17104 \\
\hline NT & 144701 & 311 & 8144 & 210241 & 763 & 27040 \\
\hline WT & 40067 & 142 & 2941 & 48449 & 1227 & 7890 \\
\hline
\end{tabular}

charity showing an interactive presence on the site, which emanates from the discussion above about this being a vital part of building relationships $[8,18]$. The following hypotheses are proposed, focusing on the conversational responses to posts by an audience:

H2a There is a significant difference between the number of comments per supporter on charities' posts on Facebook and Twitter.

H2b There is a significant difference between the number of supporters who comment on the charities' posts on Facebook and Twitter.

H2c If conversations between the charity and an engaged audience are occurring on each of the two sites, then on days when the charity posts more messages, the audience will also post more messages on the same site.

Finally, in order to investigate RQ3, based on a finding in [13] that suggested charities thought posts containing pictures were particularly 'successful', it is hypothesised that:

H3a On each site, posts containing pictures will on average produce more comments than any other post format.

By investigating $\mathrm{H} 3 \mathrm{a}$, it is hoped to be able to suggest strategies that will work most effectively for charities that wish to develop engagement and relationships with their supporters.

\subsection{Dataset}

A sample of 7 charities was used for this study, the 5 from the authors' previous study [13] (Diabetes UK, The Dogs Trust, Help for Heroes, Jeans for Genes, The 
Woodland Trust), along with two more: The National Trust and Wessex Heartbeat. The sample ensured that charities of various sizes (regarding their income) were chosen, and 6 of the 7 charities have been interviewed to ensure their views towards social media and their intended uses for it are known beforehand. For each charity, a dataset of 6 -months worth of data was collected for each site: from Twitter, a variation of the University of Southampton Tweet Harvester ${ }^{1}$ was used to collect tweets over the course of the study, whereas the Facebook dataset was collected retrospectively using a combination of the Facebook Graph API and Facebook FQL. For both sites, the collected data covered the period June-December 2013. The Twitter dataset consisted of any tweet sent by the charity, to the charity, or mentioning the charity, while from Facebook every post and associated comment made on the charity's page was collected (including posts by a supporter directly on to the page and their resulting comments). For each conversation the root post ID, the root post format, conversation chain length, number of conversation participants, whether or not the charity started the conversation, and whether or not the charity replied in the conversation were recored. While this was trivial for analysing responses to Facebook posts by simply collecting and analysing the list of comments, the process was more complex for Twitter and as such an algorithm based on the work in [5] was produced to form tweets into conversation chains.

Additionally, each user who participated in a conversation was recorded along with how many posts they made in the 6 month period, and how many conversations these fell in to. In addition, for each charity the follower count (on Twitter, as of 5th February 2014) and the number of page likes (for Facebook, as of 4th February 2014) were collected to enable proportional calculations to be carried out. A summary of the dataset is provided in Table 1. In total, 493328 posts (root posts, comments and replies) were analysed, from 201699 users.

The top 5 posts sorted by number of comments from each network for each charity were extracted so that qualitative content analysis could be carried out in order to determine whether there were any charity-specific or overall themes that appeared to cause the highest levels of conversation. This would assist in making any recommendations to the charities about what type of content produces the most desirable results, and is essential in order to contextualise the statistical work that will be used to assess the hypotheses above.

\section{Results}

\subsection{Commenter Analysis}

For RQ1, it was necessary to examine the behaviours of commenters on both sites towards each charity. Calculations were made to assess how many posts each user made, with the results shown in Table 2. A Wilcoxon signed-rank test was then carried out on these values to determine whether one site produced significantly higher values. With Twitter observably higher in each case, the test indicated

\footnotetext{
${ }^{1}$ http://tweets.soton.ac.uk
} 
Table 2: Commenter Statistics

\begin{tabular}{|l|l|l|}
\hline Charity & $\begin{array}{l}\text { Average Posts Per Com- } \\
\text { menter (FB) }\end{array}$ & $\begin{array}{l}\text { Average Posts Per Com- } \\
\text { menter (TW) }\end{array}$ \\
\hline DUK & 2.66904 & 2.98087 \\
\hline DT & 2.27573 & 2.33973 \\
\hline WH & 1.82853 & 4.90465 \\
\hline HfH & 1.65227 & 2.04518 \\
\hline JfG & 1.72773 & 3.19667 \\
\hline NT & 2.51088 & 3.19667 \\
\hline WT & 1.86335 & 2.26464 \\
\hline
\end{tabular}

that there was a difference $(\mathrm{z}=-2.366, \mathrm{p}<0.05, \mathrm{r}=-0.63)$. To examine this area further, the number of commenters who posted more than once (repeated engagers) and 6 or more times (once per month) were calculated. These values are displayed in Table 3. Using the monthly observers values (and acknowledging that this does not necessarily mean one post per month was made - a single conversation of 6 posts could also place a user into this category), a Wilcoxon signed-rank test was again carried out showing that there is a difference between the two sites in favour of Twitter $(\mathrm{z}=-2.197, \mathrm{p}<0.05, \mathrm{r}=-0.59)$. For repeat engagers the same calculation showed that there was no significant difference between the sites $(\mathrm{z}=-1.352, \mathrm{p}>0.05, \mathrm{r}=-0.36)$. This section of analysis therefore indicates that per interacting supporter, more interactions are made on Twitter than Facebook, which therefore supports H1a.

An 'engagement index' was then made for each charity on each site. On Facebook this was the average of Z-scores for the number of commenters per likes $(\mathrm{M}=0.055, \mathrm{SD}=0.026)$, the average number of conversations each user participated in $(\mathrm{M}=1.776, \mathrm{SD}=0.258)$ and the average number of posts made by each user per conversation $(\mathrm{M}=1.163, \mathrm{SD}=0.084)$. On Twitter these were the number of posters per followers $(\mathrm{M}=0.388, \mathrm{SD}=0.464)$, the average number of conversations $(\mathrm{M}=2.668, \mathrm{SD}=0.818)$ and the average number of tweets made by each user in each conversation $(\mathrm{M}=1.062, \mathrm{SD}=0.050)$. Creating $\mathrm{Z}$-scores for each charity's own score in relation to these, and averaging them provided an index for each site. To test whether charities with more engaged users on one site also had more engaged users on the other, a Spearman correlation was calculated on these values, and showed no significant correlation $(\mathrm{r}=0.321, \mathrm{p}=0.482)$, therefore H1b was not supported.

\subsection{Audience Response Analysis}

RQ2 focused more on how the audience responded to the charities' posts on each network. Looking at the data from the perspective of the posts, rather than the posters, calculations were made to find the number of comments or replies per charity-authored post on the two networks. Again, a Wilcoxon signed-rank tests 
Table 3: Repeated Engager Statistics (out of supporters who have commented at least once)

\begin{tabular}{|l|l|l|l|l|}
\hline & \multicolumn{2}{|c|}{ Repeat Engagers } & \multicolumn{2}{c|}{ Monthly Engagers } \\
\hline Charity & FB & TW & FB & TW \\
\hline DUK & $1159(38.5 \%)$ & $4134(36 \%)$ & $207(6.9 \%)$ & $1000(8.7 \%)$ \\
\hline DT & $5091(32.2 \%)$ & $6182(29.3 \%)$ & $884(5.6 \%)$ & $1152(5.5 \%)$ \\
\hline WH & $10(37.0 \%)$ & $59(33 \%)$ & $2(7.4 \%)$ & $14(7.8 \%)$ \\
\hline HfH & $1894(25.7 \%)$ & $16922(20.6 \%)$ & $180(2.4 \%)$ & $3308(4 \%)$ \\
\hline JfG & $130(26.3 \%)$ & $4637(27.1 \%)$ & $10(2 \%)$ & $769(4.5 \%)$ \\
\hline NT & $2896(35.6 \%)$ & $9904(36.6 \%)$ & $545(6.7 \%)$ & $2205(8.2 \%)$ \\
\hline WT & $916(31.1 \%)$ & $2462(31.2 \%)$ & $129(4.4 \%)$ & $427(5.4 \%)$ \\
\hline
\end{tabular}

Table 4: Post Replies Statistics (out of all supporters (likes or followers))

\begin{tabular}{|l|l|l|l|l|}
\hline & \multicolumn{2}{|l|}{$\begin{array}{l}\text { Average Responses Per } \\
\text { Post Per Supporter }\end{array}$} & \multicolumn{2}{|l|}{$\begin{array}{l}\text { Average Responders Per } \\
\text { Post Per Supporter }\end{array}$} \\
\hline Charity & FB & TW & FB & TW \\
\hline DUK & 0.00055 & 0.0005 & 0.00042 & 0.00002 \\
\hline DT & 0.00010 & 0.00001 & 0.00009 & 0.00001 \\
\hline WH & 0.00077 & 0.00029 & 0.00062 & 0.00021 \\
\hline HfH & 0.00010 & 0.00000 & 0.00009 & 0.00000 \\
\hline JfG & 0.00050 & 0.00002 & 0.00042 & 0.00001 \\
\hline NT & 0.00033 & 0.00003 & 0.00022 & 0.00001 \\
\hline WT & 0.00138 & 0.00002 & 0.00126 & 0.00002 \\
\hline
\end{tabular}

were carried out on the results. Firstly on the average number of comments per post per like (or follower), which this time showed Facebook as being consistently higher $(\mathrm{z}=-2.366, \mathrm{p}<0.05, \mathrm{r}=-0.63)$, meaning that per supporter on each site, Facebook produced a higher number of comments or replies on each of the charities's posts than Twitter, and supported H2a. This was shown again when looking at the average number of commenters or posters per like or follower on each site, with Facebook again consistently higher $(\mathrm{z}=-2.366, \mathrm{p}<0.05, \mathrm{r}=-0.63)$. This data is summarised in Table 4 and provides an indication that Facebook provides a higher proportion of interacting or engaged supporters than Twitter, supporting $\mathrm{H} 2 \mathrm{~b}$.

Finally, to test H2c, a Spearman correlation was calculated as a rudimentary analysis of the timestamps of charity posts and audience posts. For each day in the 6 month study period, a tally of how many posts were made by charity and audience was gathered. Table 5 shows that for each charity there is a significant correlation between when the charity itself posts, and when their audience posts. While H2c is supported, it is clear that the strengths of the correlations are varied and many comments may well be being made in an unsolicited way. When run 
Table 5: Charity and Supporter Post Date Correlation

\begin{tabular}{|l|l|l|}
\hline Charity & FB Correlation & TW Correlation \\
\hline DUK & $\mathrm{r}=0.643 \mathrm{p}<0.01$ & $\mathrm{r}=0.6 \mathrm{p}<0.01$ \\
\hline DT & $\mathrm{r}=0.413 \mathrm{p}<0.01$ & $\mathrm{r}=0.718 \mathrm{p}<0.01$ \\
\hline WH & $\mathrm{r}=0.341 \mathrm{p}<0.01$ & $\mathrm{r}=0.269 \mathrm{p}<0.01$ \\
\hline HfH & $\mathrm{r}=0.522 \mathrm{p}<0.01$ & $\mathrm{r}=0.302 \mathrm{p}<0.01$ \\
\hline JfG & $\mathrm{r}=0.54 \mathrm{p}<0.01$ & $\mathrm{r}=0.662 \mathrm{p}<0.01$ \\
\hline NT & $\mathrm{r}=0.541 \mathrm{p}<0.01$ & $\mathrm{r}=0.53 \mathrm{p}<0.01$ \\
\hline WT & $\mathrm{r}=0.666 \mathrm{p}<0.01$ & $\mathrm{r}=0.621 \mathrm{p}<0.01$ \\
\hline
\end{tabular}

Table 6: Post Format Responses (Facebook)

\begin{tabular}{|l|l|l|l|}
\hline Format & Comments/Posts & $\begin{array}{l}\text { Commenters/ } \\
\text { Posts }\end{array}$ & ResponseIndex \\
\hline Statuses & 23.68 & 19.71 & -0.289 \\
\hline Videos & 24.43 & 21.69 & -0.163 \\
\hline Pictures & 43.74 & 37.62 & 1.406 \\
\hline Links & 15.36 & 13.07 & -0.954 \\
\hline
\end{tabular}

through the Wilcoxon signed-rank test, these correlations showed that there was no significant difference between the strengths in correlation between each site $(\mathrm{z}=-0.338, \mathrm{p}>0.05, \mathrm{r}=-0.09)$.

\subsection{Post Format Analysis}

The quantities of each different post type (statuses, pictures, videos and links) sent by the charity were counted on the two sites. For each, the average number of comments per post (FB: $\mathrm{M}=26.806, \mathrm{SD}=12.012$, TW: $\mathrm{M}=1.496, \mathrm{SD}=0.873$ ) and the average number of commenters per post (FB: $\mathrm{M}=23.020, \mathrm{SD}=10.406$, TW: $\mathrm{M}=0.966, \mathrm{SD}=0.571$ ) were calculated (across the entire dataset for each site), with Z-scores calculated on each. An index for the engagement with each post format was then calculated as the average of these two values (Table 6 and Table 7). On Twitter, no posts were returned labelled as containing video content, so this row was excluded from the calculation. Posts containing pictures were shown to be the most engaging from a conversational point of view on both sites, receiving far higher amounts of commenters, from more commenters, than any other format. Particularly noteworthy was that on Facebook, a picture posted by a charity received on average over 37 unique commenters - on Twitter this was only 1.56 commenters per post. These results help to support H3a, and can be used to provide a strong recommendation to charities looking to increase the amount of comments that they receive. 
Table 7: Post Format Responses (Twitter)

\begin{tabular}{|l|l|l|l|}
\hline Format & Replies/Posts & Repliers/Posts & ResponseIndex \\
\hline Statuses & 0.64 & 0.423 & -0.966 \\
\hline Videos & $\mathrm{N} / \mathrm{A}$ & $\mathrm{N} / \mathrm{A}$ & $\mathrm{N} / \mathrm{A}$ \\
\hline Pictures & 2.38 & 1.56 & 1.030 \\
\hline Links & 1.46 & 0.91 & -0.065 \\
\hline
\end{tabular}

\subsection{Top Post Content Analysis}

From looking qualitatively at the content of the messages that received the highest number of comments, several recurring types of message were discovered. On Facebook, posts asking an informal question to the fans of the charity's page were common (12/35 posts), as were those promoting a competition (10/35). 7 of the posts directly referred to pictures included in them, and 21 of the 35 were classed as being informational messages for a variety of purposes (such as linking to relevant content or reporting media attention). Informally toned messages $(10 / 35)$ were more common than any formal or authoritative messages (combined 4/37). On Twitter, informal questions were again a popular type of content to be commented upon (16/35), and informational messages $(14 / 35)$ also tended to be replied to. Promoting a competition (4/35) in messages were less commonly popular than on Facebook, but general messages of thanks (4/35) indicated that these types of tweet gained a fair amount of replies. In two cases on both Facebook and Twitter, informal questions made up the majority $(5 / 5,5 / 5$, and $4 / 5,4 / 5)$ of all the charities' most popular tweets. There are indications here that informal questions are particularly effective at generating responses from the audience.

\section{Discussion}

It is interesting to discover that for the sample of charities in this study, Twitter appeared to accomodate supporters who made more interactions each, compared to Facebook (H1a). Yet when looking at the data from the point of view of responses to the charities' own posts and in relation to the number of likes or followers each charity possessed, Facebook posts received more comments (H2a), and more commenters $(\mathrm{H} 2 \mathrm{~b})$ than Twitter. It is possible that the disparity arises from H1a and could be down to the nature by which the data was collected: while the Twitter Search API allowed any messages mentioning the charity to be gathered, the Facebook data collection was restricted to what appeared on their page only, and so reflects the observable interacting users (as $\mathrm{H} 2 \mathrm{a}$ and $\mathrm{H} 2 \mathrm{~b}$ focused on responses to the charities' posts, this is not an issue). However, with the publicity of conversations such as these one of the major advantages of social media, this suggests a great opportunity on Twitter to discover these unsolicited discussions and for the charity to then take advantage. 
There is clearly a significant amount of discussion about charities occurring on Twitter, so why are the values in Tables 4, 6 and 7 so much smaller than for Facebook? Returning to the literature discussed earlier, it is possible that this is symptomatic of the 'wrong' types of messages being sent by the charitiesmessages that are not conducive to conversation, and are one-way broadcast messages instead [19], [9]. With pictures appearing to be the post format that produces the most comments in response (H3a), it is interesting to note that pictures accounted for nearly $76 \%$ of the charities' posts on Facebook, while on Twitter it was a little under $14 \%$.

Alternatively, the difference in post response rate could be down to the fact that charities do not see Twitter as a channel for relationship building in the way that they do with Facebook, supporting the views presented in the current authors' previous study [13]. It is important to note that this does currently appear to be the case, and their actual use does correlate with their perceived intentions and beliefs. If charities do see Twitter as more of a mechanism for promoting awareness and spreading information - as claimed in [13] - then signs of conversation in response to their posts would be less apparent. However it appears from the qualitative aspects of this study that there is some evidence to suggest that tweets attempting to elicit a reaction - primarily asking informal questions - are still the most popular on Twitter in terms of replies received, and engagement in this way is still possible. However a more in-depth examination of content-type and popularity is required to verify this further.

At this point, another interesting question arises. What signifies a stronger relationship: a supporter repeatedly replying to a charity's post, or a supporter regularly posting messages without a prompt from the charity itself? This is a key question to move forward in this area, requiring further study of the types of message being sent. Something is occurring which statistics are failing to account for, suggesting more in-depth qualitative methods are required now that a general understanding of the area has been obtained. The suggestion, however, is that there is a disparity of value between Facebook and Twitter-while Facebook may by better for developing relationships with continually interested supporters, Twitter's value may be in harbouring unsolicited mentions of what a wider range of people choose to do for the charity, which the charity itself can utilise for promotion. A more detailed time-series analysis of this area could provide additional understanding of this relationship.

Insights are gained from the unsupported hypothesis, H1b, which stated that charities with more engaged supporters on one site would have more engaged supporters on the other. The correlation showed that this was not supported, and suggests that engaging users on one site may not be symptomatic of a preengaged and more active audience - if this was so then both sites would tend to receive high levels of engagement compared to charities that did not. Instead, it suggests that something the charity is doing on one of the sites is probably 'working' more than on the other to stimulate conversation-again this comes down to what they look to get out of each, and requires further research to establish the overall state of a community spanning multiple social networks. 


\subsection{Limitations and Summary of Contribution}

This paper examined social media interactions around 7 UK charities on Facebook and Twitter. This is a small sample and caution must be taken before inferring the indications to the wider population. There was also limited qualitative analysis carried out which could provide much richer insights in to what people are actually conversing about. Only textual interactions were analysed, and this was only examined from the perspective of relationship building - there are other aims that are also important to charities.

The findings of this study suggest that the ways in which supporters engage with charities differs between sites, supporting the perceptions of the charities themselves presented in [13]. There is evidence to back proposals that Facebook could be better for posting messages to encourage a known supporter-base to respond, whereas Twitter appears to be a more valuable for discovering unsolicited mentions and accounts of support from any users on the network. Whether this is the cause of, or effect of, the results of studies such as [19] that claim charities in the USA focus on sending one-way messages rather than encouraging conversation is yet to be discovered. The qualitative part of this study showed that informal questions were regularly the most replied to type of tweet, and this gives some indication that the claim of 'wrong use' can not be entirely supported.

While the proportions of commenters compared to the number of supporters is low, the proportion of users posting more than once in 6 months is encouraging. It is to be expected, given the literature review, that the majority of the community will be content to listen. These results help to highlight how valuable small portions of an audience are in creating a large amount of conversation around a topic - and the techniques used to locate these users can now be taken further to assess their overall contribution to the buzz around the charity.

Based on the discoveries in this paper, we can make some suggestions to charities wishing to develop their relationship building strategies on social media. Facebook appears to be the more suitable choice for generating discussions about relevant topics with the dedicated, committed supporter base. Twitter seems to hold great potential value for finding the extra, satellite discussions about the charities, and then supporting and amplifying these using Twitter's ability to quickly disseminate and spread messages. On both sites, pictures should be used when the charity desires a conversation or many replies. It seems apparent, however, that a statistical approach such as this to analysing social media can only say so much, despite providing initial insights and an overall picture of this area. Further qualitative analysis of messages is required, and this must be used in conjunction with the aims and view of the charities discovered in [13] to determine further where the value in social media truly arises from.

\section{Acknowledgments}

This research was funded by the Research Councils UK Digital Economy Programme, Web Science Doctoral Training Centre, University of Southampton. $\mathrm{EP} / \mathrm{G} 036926 / 1$. 


\section{References}

[1] Bernstein, M.S., Bakshy, E., Burke, M., Karrer, B.: Quantifying the invisible audience in social networks. In: Proceedings of the SIGCHI Conference on Human Factors in Computing Systems. pp. 21-30. CHI '13, ACM, New York, NY, USA (2013), http://doi.acm.org/10.1145/2470654.2470658

[2] Boyd, D., Golder, S., Lotan, G.: Tweet, tweet, retweet: Conversational aspects of retweeting on twitter. In: System Sciences (HICSS), 2010 43rd Hawaii International Conference on. pp. 1-10 (2010)

[3] Briones, R.L., Kuch, B., Liu, B.F., Jin, Y.: Keeping up with the digital age: How the american red cross uses social media to build relationships. Public Relations Review 37(1), 37-43 (Mar 2011), http://www. sciencedirect. $\mathrm{com} / \mathrm{science/article/pii/S0363811110001335}$

[4] Cho, M., Schweickart, T., Haase, A.: Public engagement with nonprofit organizations on facebook. Public Relations Review 40(3), 565-567 (September 2014), http://www.sciencedirect.com/science/article/ $\mathrm{pii} / \mathrm{S} 0363811114000241$

[5] Cogan, P., Andrews, M., Bradonjic, M., Kennedy, W.S., Sala, A., Tucci, G.: Reconstruction and analysis of twitter conversation graphs. In: Proceedings of the First ACM International Workshop on Hot Topics on Interdisciplinary Social Networks Research. pp. 25-31. HotSocial '12, ACM, New York, NY, USA (2012), http://doi.acm.org/10.1145/2392622.2392626

[6] Crawford, K.: Following you: Disciplines of listening in social media. Continuum 23(4), 525-535 (2009), http://www.tandfonline.com/doi/abs/10. $1080 / 10304310903003270$

[7] Cvijikj, I.P., Michahelles, F.: Understanding the user generated content and interactions on a facebook brand page. International Journal of Social and Humanistic Computing 2(1), 118-140 (Jan 2013), http://dx.doi.org/10. 1504/I JSHC . 2013.053270

[8] Jo, S., Kim, Y.: The effect of web characteristics on relationship building. Journal of Public Relations Research 15(3), 199-223 (2003), http://www. tandf online.com/doi/abs/10.1207/S1532754XJPRR1503_1

[9] Lovejoy, K., Saxton, G.D.: Information, community, and action: How nonprofit organizations use social media. Journal of Computer-Mediated Communication 17(3), 337-353 (2012), http://onlinelibrary.wiley.com/ doi/10.1111/j.1083-6101.2012.01576.x/abstract

[10] Nielsen, J.: Participation inequality: Encouraging more users to contribute. http://www useit.com/alertbox/participation_inequality. html [Last Accessed: 26 Nov 2014] (Oct 2006), http://www.useit.com/ alertbox/participation_inequality.html

[11] Paek, H.J., Hove, T., Jung, Y., Cole, R.T.: Engagement across three social media platforms: An exploratory study of a cause-related PR campaign. Public Relations Review 39(5), 526-533 (Dec 2013), http://www . sciencedirect.com/science/article/pii/S0363811113001379 
[12] Phethean, C., Tiropanis, T., Harris, L.: Measuring the performance of social media marketing in the charitable domain. In: Web Science 2012. Evanston, IL, USA (June 2012)

[13] Phethean, C., Tiropanis, T., Harris, L.: Rethinking measurements of social media use by charities: A mixed methods approach. In: Web Science 2013. ACM, Paris, France (May 2013)

[14] Rotman, D., Vieweg, S., Yardi, S., Chi, E., Preece, J., Shneiderman, B., Pirolli, P., Glaisyer, T.: From slacktivism to activism: participatory culture in the age of social media. In: Conference on Human Factors in Computing Systems. ACM, Vancouver BC (May 2011), http://dl.acm.org/ citation. .fm?id=1979543

[15] Smith, A.N., Fischer, E., Yongjian, C.: How does brand-related usergenerated content differ across YouTube, Facebook, and Twitter? Journal of Interactive Marketing 26(2), 102-113 (May 2012), http://www. sciencedirect.com/science/article/pii/S1094996812000059

[16] Takahashi, M., Fujimoto, M., Yamasaki, N.: The active lurker: influence of an in-house online community on its outside environment. In: GROUP '03 Proceedings of the 2003 international ACM SIGGROUP conference on Supporting group work. ACM Press (2003), http://portal.acm.org/ citation. $\mathrm{cfm}$ ?doid $=958160.958162$

[17] Tinati, R., Carr, L., Hall, W., Bentwood, J.: Identifying communicator roles in twitter. In: Proceedings of the 21st international conference companion on World Wide Web. pp. 1161-1168. WWW '12 Companion, ACM, New York, NY, USA (2012), http://doi.acm.org/10.1145/2187980.2188256

[18] Waters, R.D., Burnett, E., Lamm, A., Lucas, J.: Engaging stakeholders through social networking: How nonprofit organizations are using facebook. Public Relations Review 35(2), 102-106 (Jun 2009), http://linkinghub. elsevier.com/retrieve/pii/S0363811109000046

[19] Waters, R.D., Jamal, J.Y.: Tweet, tweet, tweet: A content analysis of nonprofit organizations' twitter updates. Public Relations Review 37(3), 321324 (Sep 2011), http://www.sciencedirect.com/science/article/pii/ S0363811111000361 